\title{
Registros de ensaios clínicos e as consequências para as publicações científicas
}

\section{Clinical trials registers and their implications for scientific publication}

\author{
Regina Celia Figueiredo Castro
}

\begin{abstract}
RESUMO
O presente trabalho apresenta as recomendações do International Committee of Medical Journal Editors (ICMJE) e da Organização Mundial da Saúde (OMS) para os registros de ensaios clínicos. Discute as consequências para as publicações científicas e o papel da BIREME para a inclusão da América Latina e Caribe nessas iniciativas. Descreve o portal de acesso à Plataforma Internacional de Registro de Ensaios Clínicos (ICTRP), coordenada pela OMS, e as principais iniciativas existentes, destacando as da América Latina e Caribe.
\end{abstract}

Palavras-chave: Ensaio Clínico. Publicações Periódicas. Sistema de Registros/Ensaio Clínico. Ensaios Clínicos como Assunto.

\section{Introdução}

Ao realizar ensaios clínicos os pesquisadores se responsabilizam pela divulgação dos resultados, tanto para a comunidade científica como para os participantes dessas pesquisas. Segundo a ética da publicação científica os resultados de pesquisas devem ser divulgados, sejam eles positivos ou negativos, principalmente naquelas que envolvem seres humanos. No entanto, os editores de revistas médicas e pesquisadores perceberam que na literatura da área médica o número de artigos que relatavam resultados negativos era baixo, ou quando publicados, esses artigos nem sempre relatavam todos os resultados.

Sendo assim, verificou-se a necessidade de manter registro das pesquisas clínicas em andamento por vários motivos: para aumentar a transparência das pesquisas científicas tanto para a comunidade científica como para o público em geral; pela responsabilidade ética com os participantes dos estudos clínicos; para divulgar publicamente as pesquisas em andamento e seus resultados, sejam estes positivos ou negativos; para dirimir e evitar conflitos de interesses, principalmente em relação ao financiamento das pesquisas.

Dada a importância dos registros dos ensaios clínicos, em 2004, o International Committee of Medical Journal Editors (ICMJE), (também conhecido como Grupo de Vancouver), que é constituído por editores das principais revistas médicas e por representante da Biblioteca Nacional de Medicina, dos Estados Unidos, lançou uma recomendação a todos os editores de revistas médicas ${ }^{1,2}$. O objetivo foi estimular a criação de uma base de dados pública de registros de ensaios clínicos, em primeiro lugar como uma forma de reconhecer aos voluntários participantes das pesquisas clínicas e em segundo, para dar conhecimento público aos resultados dessas pesquisas.

Em 2004, a Declaração do México, resultado da Cúpula Ministerial de Pesquisa em Saúde, recomendou que a OMS facilitasse a criação de uma rede in- 
ternacional de registros de ensaios clínicos e um portal integrado de pesquisas. Com base nessa recomendação e como instituição internacional na área da saúde, a Organização Mundial da Saúde reforçou a recomendação do ICMJE sobre o registro de ensaios clínicos e criou a Plataforma de Registro de Ensaios Clínicos ${ }^{3}$.

Como afirmou o Dr. Timothy Evans, Diretor Assistente da OMS, na nota de imprensa lançada pela $\mathrm{OMS}^{4}$, "O registro de todos os ensaios clínicos e a disponibilidade de todas as principais informações no momento do registro serão fundamentais para assegurar a transparência na pesquisa médica e cumprir com as responsabilidades éticas para com os pacientes e participantes dos estudos".

O ICMJE, em conjunto com a OMS, definiu um conjunto de vinte campos obrigatórios ${ }^{3}$ para os registros de ensaios clínicos, dentre eles, data de realização e registro, instituições financiadoras, título da pesquisa, pesquisador responsável, tipo de estudo e de intervenção, objetivos do estudo, etc. Esses campos de dados constituem os campos mínimos que todos os Registros de Ensaios Clínicos devem respeitar para que seja possível intercambiar dados e dar a conhecer os estudos realizados e em andamento. Embora não recomendem nenhum Registro de Ensaios Clínicos em especial, o ICMJE sugere que os pesquisadores escolham aqueles que contenham todos esses campos.

Segundo a definição da Organização Mundial da Saúde 2 , "para efeitos de registro, um ensaio clínico significa qualquer pesquisa que seleciona prospectivamente participantes humanos, indivíduos ou grupos de pessoas, para participarem de intervenções relacionadas à saúde humana para avaliar efeitos e resultados na saúde. Essas intervenções incluem, mas não se restringem a pesquisas com drogas, células e outros produtos biológicos, procedimentos cirúrgicos ou radiológicos, aparelhos, tratamentos terapêuticos, mudanças no processo de atenção, cuidados preventivos, etc.".

\section{Implicações para as publicações científicas}

Na recomendação do $\mathrm{ICMJE}^{2}$, o Grupo solicita que os editores aceitem como condição prévia à publicação, apenas os artigos sobre ensaios clínicos que tenham sido registrados em uma base de dados de Registro de Ensaios Clínicos. Os registros devem ser feitos pelos pesquisadores antes de iniciar o ensaio e de selecionar os participantes da pesquisa. A data estabelecida pelo ICMJE para adoção dessa política edi- torial foi para estudos iniciados em julho de 2005, e para estudos em andamento registrados antes de setembro de 2005. Editores de revistas médicas de países desenvolvidos apoiaram imediatamente a recomendação do ICMJE, começando pelas que integram esse grupo.

Para dar cumprimento a essa recomendação da OMS nos países da América Latina e Caribe, o Centro Latino-Americano de Informação em Ciências da Saúde (BIREME/OPAS/OMS) também lançou uma recomendação ${ }^{5}$ aos editores de revistas indexadas nas bases de dados LILACS (Literatura Latino-Americana e do Caribe em Ciências da Saúde) e SciELO (Scientific Electronic Library Online), em maio de 2007. Nessa recomendação, a BIREME estabelece que todos os editores de revistas médicas latino-americanas exijam o número de registro de artigos com resultados de ensaios clínicos, como um critério para a aceitação dos trabalhos.

Seguindo o modelo proposto pelo ICMJE, a BIREME sugere um texto para que os editores incluam nas Instruções aos Autores de suas revistas, orientando os autores sobre a necessidade de registrar suas pesquisas clínicas. A atualização das Instruções aos Autores nas revistas médicas indexadas na SciELO tem sido acompanhada pela BIREME, como um controle sobre a adoção dessa recomendação. Da mesma forma, a BIREME tem acompanhado a indicação pelos autores e editores do número de registro nos artigos sobre ensaios clínicos. Até julho de 2008, nenhum ensaio clínico havia sido publicado em revistas latino-americanas.

\section{Plataforma de Reglstro de Ensalos Clínicos}

A Plataforma de Registro de Ensaios Clínicos (International Clinical Trials Registry Platform ICTRP), coordenada pela OMS, dispõe de um portal de pesquisa (Figura 1) aberto ao público em geral que permite a pesquisa integrada em todas as bases de dados certificadas pela OMS e incluídas na platafor$\mathrm{ma}^{6}$.

Na modalidade de pesquisa avançada, o usuário pode buscar por qualquer palavra do título, pela condição clínica, pelo tipo de intervenção, pelo estado do recrutamento, patrocinadores das pesquisas. É possível selecionar os países de realização dos estudos, delimitar datas e definir se a busca será feita em um registro específico ou aplicá-la a todos os registros (Figura 2). 
INTERNATIONAL CLINICAL TRIALS REGISTRY PLATFORM SEARCH PORTAL

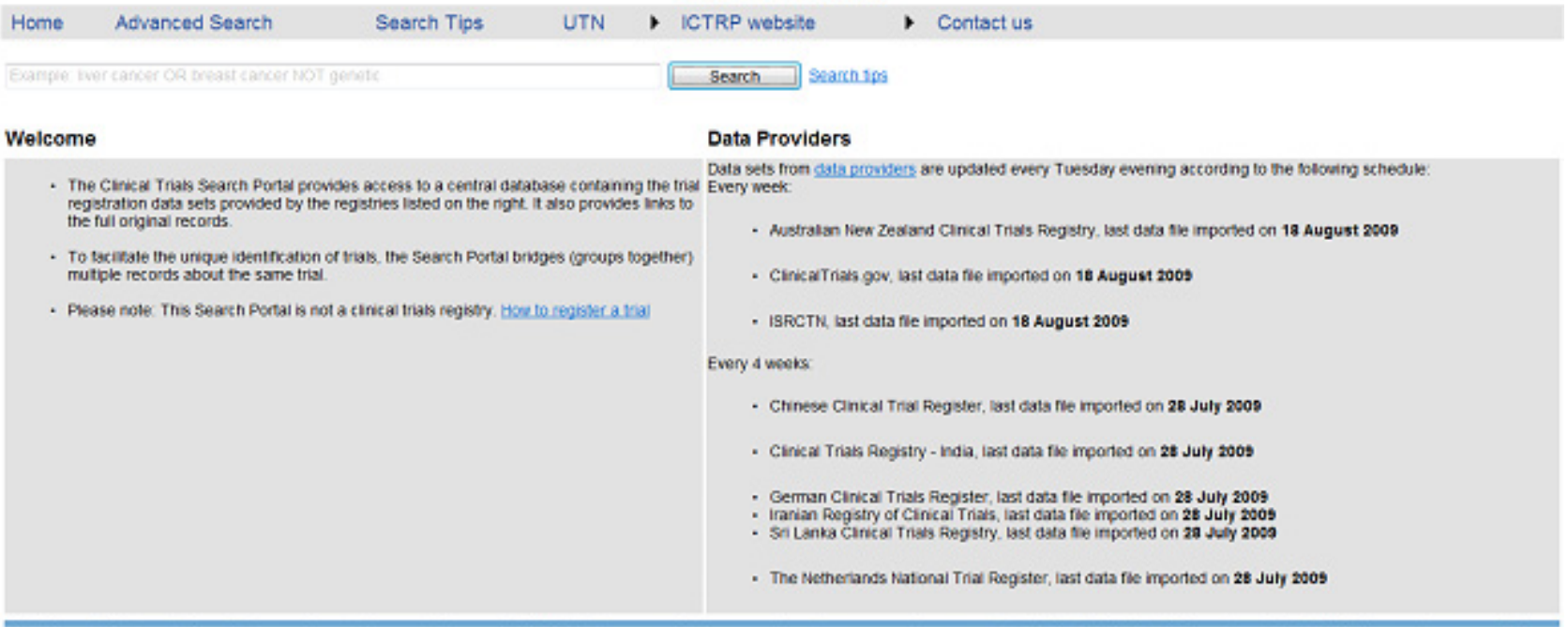

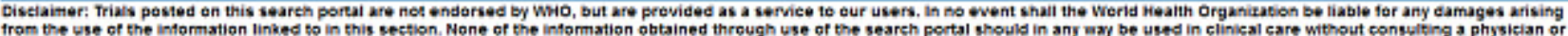
from the wse of the intormation linked to in this section. None of the information obtained through use of the seareh portal Nhould in any way be used in clinical care without consalting a physielan of licensed health protessional. who is not respensible tor the arouracy, completeness andilor use made of the content displayed for any trial record.

\section{Cepyright - World Heath Crganization - Version 3.1 - Yersion histen}

Figura 1- Portal de pesquisa da Plataforma Internacional de Registros de Ensaios Clínicos, disponível em: http://www.who.int/trialsearch/

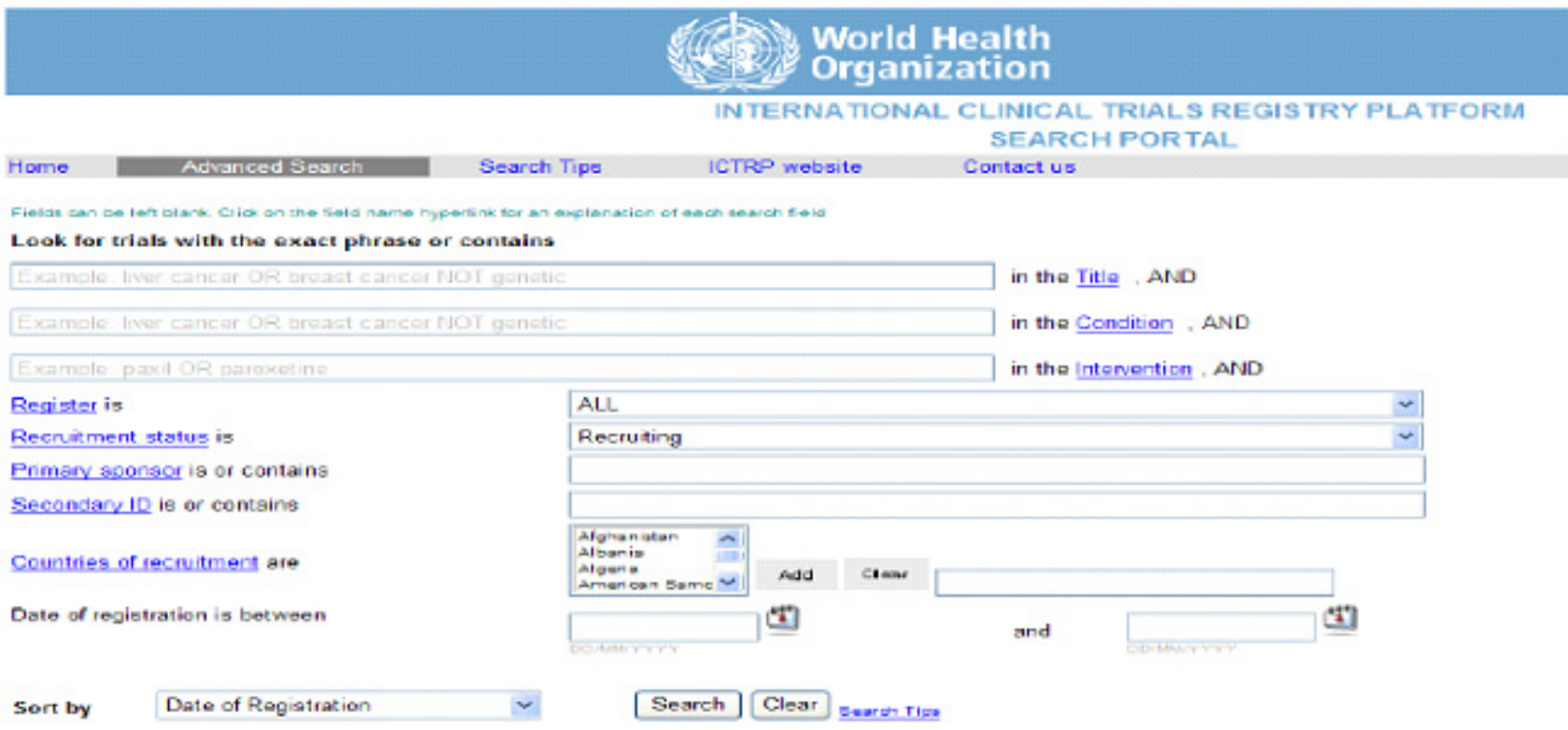

Figura 2- Módulo de pesquisa avançada, Portal de Pesquisas, Plataforma Internacional de Registros de Ensaios Clínicos, OMS (http://www.who.int/trialsearch/AdvSearch.aspx) 


\section{Iniciativas existentes}

Várias iniciativas para a criação de Registros de Ensaios Clínicos nacionais ou de áreas de intervenção específicas foram desenvolvidas em todo o mundo ${ }^{7,8}$. A OMS certifica e classifica esses Registros de acordo com o cumprimento dos campos mínimos definidos pelo ICMJE. Os Registros que não cumprem todos os critérios devem associar-se aos Registros Primários, que foram certificados, e devem garantir a inclusão de toda a informação necessária.

Até julho de 2008, as iniciativas existentes eram:

- Australian New Zealand Clinical Trials Registry (ANZCTR)

- Chinese Clinical Trial Register (ChiCTR)

- ClinicalTrials.gov (Estados Unidos)

- Clinical Trials Registry - India (CTRI)

- ISRCTN.org (Inglaterra)

- The Netherlands National Trial Register (NTR)

- Sri Lanka Clinical Trials Registry (SLCTR)

Em novembro de 2008, são dez os Registros atualizados no Portal de Pesquisa. Além dos já mencionados, foram incluídos:

- German Clinical Trials Register (DRKS)

- Iranian Registry of Clinical Trials (IRCT)

- Japan Primary Registries Network

Além dos 20 campos básicos e obrigatórios exigidos pela OMS, vários campos adicionais foram criados pelos distintos Registros. Alguns são realmente campos adicionais, criados principalmente para atender a exigências nacionais para o registro de pesquisas clínicas. Entretanto, alguns Registros adotam o mesmo nome de campo, mas com semântica diferente, ou adotam nomes diferentes para o mesmo conteúdo semântico recomendado pela OMS ou ainda subdividem o conteúdo dos campos básicos em diferentes níveis hierárquicos (gerais ou específicos). Exemplos de campos adicionais: fontes de financiamento, aprovação por Comitê de Ética em Pesquisa, metodologia do estudo, descrição das amostras biológicas e da população em estudo, palavras-chave, dentre outros. Segundo análise realizada pela BIREME sobre as iniciativas existentes, o Registro que tem mais campos específicos ou detalhamento de campos obrigatórios é o ClinicalTrials.gov, dos Estados Unidos.

Na América Latina, a Organização Pan-Americana da Saúde (OPAS) divulgou um diagnóstico da situação ${ }^{9}$ e tem apoiado a criação de uma Plataforma regional para a América Latina e Caribe de Registros de Ensaios Clínicos, em conjunto com a BIREME. A proposta de criação dessa plataforma foi apresentada durante o VIII Congresso Brasileiro de Saúde Coletiva no Rio de Janeiro, em 2006 e novamente discutida com representantes da Argentina, Chile, Cuba, Colômbia e Brasil, durante o XV Colóquio Cochrane, realizado em São Paulo, em outubro de 2007.

A criação da Plataforma Regional para a América Latina e Caribe permitirá a criação de registros nacionais e de um portal de busca integrada nos registros existentes na Região. Os países que não têm sistemas próprios poderão adotar o modelo proposto para a criação de seus registros nacionais. Até julho de 2008, existiam os seguintes registros:

- LatinRec (Latin-American Clinical Trials Registry), Colombia ${ }^{10}$

- Registro Público Cubano de Ensayos Clínicos, Cuba $^{11}$

No Brasil, a discussão sobre o Registro Nacional de Ensaios Clínicos, iniciada em 2006 no VIII Congresso Brasileiro de Saúde Coletiva, envolve o Departamento de Ciência e Tecnologia (Decit) da Secretaria de Ciência e Tecnologia e Insumos Estratégicos, do Ministério da Saúde, a Fundação Oswaldo Cruz, a Agência Nacional de Vigilânica Sanitária (Anvisa), e o Conselho Nacional de Ética em Pesquisa. Essas instituições, em conjunto com a BIREME e a Área de Pesquisas da OPAS, iniciaram discussões para a criação de um Registro Nacional para o Brasil. Carvalheiro e Quental ${ }^{12}$ descrevem algumas questões que têm sido discutidas no Brasil. Em julho de 2008, o Ministério da Saúde publicou Portaria ${ }^{13}$ que estabeleceu uma Comissão para Elaboração do Projeto de Implantação do Registro Brasileiro de Ensaios Clínicos - REBRAC, sendo sua finalização prevista para o ano de 2009, sob a coordenação do Decit/MS.

Iniciativas complementares também foram desenvolvidas pela BIREME na Biblioteca Virtual em Saúde (BVS), como o Portal de Evidências ${ }^{14}$ e o Portal Cochrane BVS ${ }^{15}$. O Portal de Evidências reúne, organiza e oferece acesso integrado a fontes de informação em saúde de melhor nível de evidência, de acordo com a metodologia proposta pela Medicina Baseada em Evidências (MBE) e oferece acesso a fontes de informação sobre a própria metodologia MBE. A coleção do Portal Cochrane BVS é formada pelo conjunto das oito bases da Cochrane Library; pelas bases da Biblioteca Cochrane Plus, reunidas pelo Centro Cochrane Ibero-Americano, que inclui conteúdos em espanhol; e pelos resumos de revisões sistemáticas Cochrane traduzidos ao português, produzidos pelo Centro Cochrane do Brasil. 


\section{Considerações Finais}

A necessidade de assegurar a transparência dos estudos clínicos através de sua divulgação para a comunidade científica e para os participantes é evidente e tem sido atendida pelas diversas propostas da OMS, implementadas e apoiadas pela (BIREME/ OPAS/OMS). No entanto, tão importante quanto o desenvolvimento da base de dados pública de regis- tros de ensaios clínicos é promover a sua ampla divulgação na comunidade científica, o que foi objeto desse trabalho, que expõe de forma breve, facilitada e simples, o contexto no qual a base de dados foi idealizada e tem sido atualmente implementada. Dessa forma, este texto é de caráter essencialmente informativo, visando estabelecer um contato inicial dos pesquisadores com a nova necessidade de registrar um estudo clínico.

\begin{abstract}
This document discusses the recommendations of the International Committee of Medical Journal Editors (ICMJE) and of the World Health Organization for clinical trials registering. The consequences for scientific publications and the role of BIREME for joining Latin America and Caribbean countries in these initiatives are discussed. The International Clinical Trials Registry Platform (ICTRP) coordinated by WHO as well as the existent initiatives are described, highlighting those from Latin America and the Caribbean.
\end{abstract}

Keywords: Clinical Trial. Periodicals. Registries/Clinical Trial. Clinical Trials as Topic.

\section{Referênclas}

1. De Angelis C, Drazen JM, Frizelle FA, Haug C, Hoey J, Horton $\mathrm{R}$, et al. Clinical trial registration: a statement from the International Committee of Medical Journal Editors. Ann Intern Med. [Internet]. 2004 [citado em 2008 Dez 12];141:477-8. Epub 2004 Sep 8. Disponível em: http://www.annals.org/cgi/reprint/141/ 6/477.pdf e http://www.icmje.org/clin_trial.pdf

2. International Committee of Medical Journal Editors. Is this clinical trial fully registered? A statement from the International Committee of Medical Journal Editors. ICMJE Editorial [Internet]. 2005 May [citado em 2008 Dez 12]. Disponível em: http:// www.icmje.org/clin_trialup.htm

3. International Clinical Trials Registry Platform (ICTRP). Geneva: World Health Organization [Internet] [citado em 2008 Dez 12]. Disponível em: http://www.who.int/ictrp/en/

4. World Health Organization. The World Health Organization announces new standards for registration of all human medical research. [Internet]. Geneva: 2006 May 19 [citado em 2008 Dez 12]. Disponível em: http://www.who.int/mediacentre/ news/releases/2006/pr25/en/index.html

5. Recomendação aos editores de revistas indexadas na LILACS e SciELO sobre registro de ensaios clínicos. [Internet]. São Paulo: BIREME, 2007 maio 15. [citado em 2008 Dez 12]. Disponível em: http://espacio.bvsalud.org/files/5/1/ 051111052007/recomend_edit_rev_2007\%20port.pdf

6. International Clinical Trials Registry Platform. Search Portal. [Internet]. Geneva: World Health Organization. [citado em 2008 Dez 12]. Disponível em: http://www.who.int/trialsearch/

7. International Committee of Medical Journal Editors. Clinical Trial Registration: looking back and moving ahead. ICMJE Editorial [Internet], Jun. 2008 [citado em 2008 Dez 12]. Disponível em: http://www.icmje.org/clin_trial07.pdf
8. Ghersi D, Clarke M, Berlin J, Gülmezoglu AM, Kush R, Lumbiganon $\mathrm{P}$ et al. Reporting the findings of clinical trials: a discussion paper. Bull World Health Organ [Internet] 2008 Jun [citado em 2008 Dez 12]; 86(6):492-493. Disponível em: http:/ /www.scielosp.org/scielo.php?script=sci_arttext\&pid=S0042$96862008000600021 \&$ lng $=p t \& n r m=i s o$.

9. Cuervo LG, Valdes A, Clark ML. El registro internacional de ensayos clínicos. Rev Panam Salud Publica [Internet], jun. 2006 [citado em 2008 Dez 12]; 19(6):365-70. Disponível em: http://www.scielosp.org/pdf/rpsp/v19n6/30516.pdf

10. Reveiz L, Delgado MB, Urrutia G et al. The Latin American Ongoing Clinical Trial Register (LATINREC). Rev Panam Salud Publica [Internet], jun. 2006 [citado em 2008 Dez 12]; 19(6):417-22. Disponível em: http://www.scielosp.org/pdf/ rpsp/v19n6/30529.pdf

11. Registro Público Cubano de Ensayos Clínicos. [Internet]. Cuba, Centro Nacional Coordinador de Ensayos Clínicos. [citado em 2008 Dez 12]. Disponível em: http://registroclinico.sld.cu/

12. Carvalheiro JR, Quental C. Registro de ensaios clínicos: a discussão internacional e os posicionamentos possíveis para o Brasil. R. Eletr. de Com. Inf. Inov. Saúde [Internet]. 2007 janjun[citado em 2008 Dez 12]; 1(1):63-9. Disponível em: http://www.reciis.cict.fiocruz.br/index.php/reciis/article/ viewFile/42/59

13. Ministério da Saúde. Portaria MS/GM No 1.345 que insitui a Comissão para Elaboração do Projeto de Implantação do Registro Brasileiro de Ensaios Clínicos REBRAC. Brasília, 2 de julho de 2008.

14. Portal de Evidências. [Internet]. São Paulo: BIREME. [citado em 2008 Dez 12]. Disponível em: http://evidences.bvsalud.org/php/index.php?lang=pt

15. Portal Cochrane BVS. São Paulo: BIREME. [Internet]. [citado em 2008 Dez 12]. Disponível em: http://cochrane.bvsalud.org/portal/php/index.php?lang=pt 